\title{
Correlation-based approach to analysis of spiking networks
}

\author{
Michael Krumin, Shy Shoham* \\ From Nineteenth Annual Computational Neuroscience Meeting: CNS*2010 \\ San Antonio, TX, USA. 24-30 July 2010
}

The correlation structure of neural activity is believed to play a major role in the encoding and possibly decoding of information in neural populations. In addition, some of the most fundamental and widely applied tools for the identification of systems rely on the use of second order statistical properties (correlation or spectral). An arsenal of tools for identifying spike train models from their correlations, rather than from their full observed realizations could thus form a welcome bridge between 'classical' signal processing ideas and tools and the field of neural spike train analysis.

Recently, several methods were developed for controlling the correlation structure of multi-channel synthetic spike trains [1,3-5], and in related work, correlation based analysis of spike trains was used for blind identification of single-neuron models [5], for identifying compact autoregressive models for multi-channel spike trains, and for facilitating their causal network analysis [6]. However, the diversity of correlation structures that can be explained by the non-recurrent generative models used in these studies is limited, and hence, methods based on such models occasionally fail while analyzing correlation structures that are observed in neural activity. Here, we extend this framework by deriving closed-form expressions for the correlation structure of a more powerful multivariate self- and mutually-exciting Hawkes model class that is driven by exogenous non-negative inputs. We demonstrate that the resulting Linear-Nonlinear-Hawkes (LNH) framework is capable of capturing the dynamics of spike trains with a generally richer multi-correlation structure. We explore several new applications of this framework including highly compact representation of multi-channel spike train data and causal analysis of network information flow.

\footnotetext{
* Correspondence: sshoham@bm.technion.ac.il

Faculty of Biomedical Engineering, Technion - Israel Institute of Technology,
} Haifa, 32000, Israel

\section{Acknowledgements}

This work was supported by Israeli Science Foundation grant \#1248/06 and European Research Council starting grant \#211055.

Published: 20 July 2010

\section{References}

1. Brette R: Generation of Correlated Spike Trains. Neural Computation 2009, 21(1):188-215.

2. Macke JH, Berens P, Ecker AS, Tolias AS, Bethge M: Generating Spike Trains with Specified Correlation Coefficients. Neural Computation 2009, 21(2):397-423.

3. Krumin M, Shoham S: Generation of Spike Trains with Controlled Autoand Cross-Correlation Functions. Neural Computation 2009, 21(6):1642-64

4. Tchumatchenko T, Malyshev A, Geisel T, Volgushev M, Wolf F: Correlations and Synchrony in Threshold Neuron Models. Phys Rev Lett 2010, 104(5):058102.

5. Krumin M, Shimron A, Shoham S: Correlation-distortion based identification of Linear-Nonlinear-Poisson models. J Comput Neurosci 2009.

6. Krumin M, Shoham S: Multivariate Auto-Regressive Modeling and Granger Causality Analysis of Multiple Spike Trains. Computational Intelligence and Neuroscience 2000.

doi:10.1186/1471-2202-11-S1-P182

Cite this article as: Krumin and Shoham: Correlation-based approach to analysis of spiking networks. BMC Neuroscience 2010 11(Suppl 1):P182.

\section{Submit your next manuscript to BioMed Central and take full advantage of: \\ - Convenient online submission \\ - Thorough peer review \\ - No space constraints or color figure charges \\ - Immediate publication on acceptance \\ - Inclusion in PubMed, CAS, Scopus and Google Scholar \\ - Research which is freely available for redistribution

